\title{
1 Stabilizing proteins through saturation suppressor
} 2 mutagenesis.

\section{Shahbaz Ahmed ${ }^{1}$, Kavyashree Manjunath ${ }^{2}$, Raghavan 4 Varadarajan ${ }^{1, *}$}

5 8

$9 \quad *$ Author for correspondence

10 Telephone: +91-80-2293-. 2612

11 Fax: +91-80-23600535

12 Email: varadar@iisc.ac.in

14 Running Title: Screening for stability

15 Number of manuscript pages: 36

16 Number of supplementary material pages: 7

17 Number of figures: 12 


\section{Abstract}

27 While there have been recent, transformative advances in the area of protein structure prediction, 28 prediction of point mutations that improve protein stability remains challenging. It is possible to 29 construct and screen large mutant libraries for improved activity or ligand binding, however reliable screens for mutants that improve protein stability do not exist, especially for proteins that are well folded and relatively stable. We demonstrate that incorporation of a single, specific destabilizing, (parent inactive) mutation into each member of a single-site saturation mutagenesis library followed by screening for suppressors, allows for robust and accurate identification of stabilizing mutations.

34 When coupled to FACS sorting of a yeast surface display library of the bacterial toxin CcdB, followed by deep sequencing of sorted populations, multiple stabilizing mutations could be identified after a single round of sorting. Multiple libraries with different parent inactive mutations could be pooled and simultaneously screened to further enhance the accuracy of identification of stabilizing mutations. Individual stabilizing mutations could be combined to result in a multimutant with increase in thermal melting temperature of about $20^{\circ} \mathrm{C}$ and enhanced tolerance to high temperature exposure. The method employs small library sizes and can be readily extended to other display and screening formats to rapidly isolate stabilized protein mutants. mutagenesis 
Introduction

Directed evolution has drastically reduced the time required to engineer desired function into proteins (Hermes et al, 1990; Lehtovaara et al, 1988; Tao \& Cornish, 2002; Turner, 2009). Enzymes and other proteins with altered function or binding specificity have been evolved using yeast surface display (YSD), phage display (Shusta et al, 2008; Wu et al, 2016) or other in vivo functional screens (Waldo et al, 1999; Wigley et al, 2001; Maxwell et al, 1999). Phage display utilizes its surface proteins pIII and pVIII, which are fused to the protein of interest (Mimmi et al, 2019). Phage display can be used to generate libraries of very high diversity (Schwimmer et al, 2013) which can be screened for binding to a target ligand. Agglutinin based Aga2p is a widely used system to display proteins on the yeast cell surface (Shusta et al, 2008). Aga2p is a small protein, covalently linked via disulphide linkages to the yeast cell surface protein Aga1(Boder \& Wittrup, 1997). Different populations in a yeast library can be enriched using FACS. Relative to the phage display library, sizes are lower $\left(\sim 10^{11}\right.$ vs $\left.10^{7}\right)$. However, with YSD, eukaryotic post translation modifications are possible. While screening for mutants with improved binding or enzymatic activity is straight forward, it is non-trivial to screen for mutants with improved stability. Some prior studies have suggested that stabilized mutants are expressed at higher levels than wild type (WT), however, several other studies have not observed this (Shusta et al, 1999; Park et al, 2006; Piatesi et al, 2006). In several cases it was observed that for stable proteins, mutants with improved stability are expressed at a similar level to WT (Esteban \& Zhao, 2004; Hagihara \& Kim, 2002; Park et al, 2006; Piatesi et al, 2006) and hence expression alone cannot be used to discriminate mutants with higher stability from mutants with a slightly destabilized phenotype. We recently showed that the amount of active protein on the yeast cell surface (detected by the amount of bound ligand) correlates better with in vitro thermal stability or in vivo solubility than the amount of total protein on the yeast cell surface, for destabilized mutants (Ahmed et al, 2021). However, mutants above a certain stability threshold show similar expression and ligand binding to wild type. An alternative approach to isolate stabilizing mutations is to introduce a destabilizing mutation hereafter 
referred to as parent inactivation mutation (PIM) and then create mutant libraries in this background to screen for suppressors (Araya et al, 2012; Blacklow et al, 1991; Brachmann et al, 1998; Foit et al, 2009; Sahoo et al, 2015). This methodology has required multiple rounds of enrichment to isolate stable mutants. The reversion of PIM to wild type or non-destabilizing mutants during library generation or enrichment enriches for mutants lacking the PIM instead of desired PIM-suppressor pairs. Additionally, in multi-round format, this methodology potentially allows isolation of only a few stabilizing mutations, and does not distinguish between allele specific and global suppressors. It is also unclear whether it will always be possible to isolate suppressors for every PIM. In the present study, we have modified this approach by introducing PIMs in the background of a single-site, saturation mutagenesis (SSM) library of bacterial toxin CcdB, sorted different populations and reconstructed the

MFI of each mutant as described (Ahmed et al, 2021). We use both the binding MFI and expression

MFI as criteria to differentiate between stabilized mutants, WT-like and destabilized mutants. Using of false positives.

\section{Results}

\section{Second site Saturation Suppressor Mutagenesis (SSSM) methodology.}

In a previous study, when an SSM library of the bacterial toxin CcdB was used to isolate stabilized mutants based on their binding to GyA14 or binding/expression ratio on the yeast cell surface, a large number of false positives were obtained (Ahmed et al, 2021). We found that if the stability of displayed protein crosses a threshold, it is difficult to identify stabilized mutants solely based on the extent of ligand binding or expression as mutants show a similar level of binding and expression on the yeast cell surface irrespective of the stability. To minimize such threshold effects, we used single-site, saturation suppressor mutagenesis (SSSM) methodology (Figure 1), as described previously (Sahoo et 
suppressors reverse the destabilizing effect of the PIM by locally compensating packing defects caused

by the PIM. Proximal suppressors are allele specific and do not show stabilizing effects as a single

mutant in the absence of the PIM (Nikolova, 2000; Sahoo et al, 2015). Distal suppressors are located

far from the PIM and often act as global suppressors, reversing the effects of multiple individual PIMs.

A distal suppressor also typically stabilizes the protein relative to WT, in the absence of the PIM.

\section{Selection of PIM and sorting of Second Site Saturation Mutagenesis (SSSM) library of CcdB}

$\mathrm{CcdB}$ is a bacterial toxin that causes bacterial cell death by binding and poisoning DNA gyrase (Bernard \& Couturier, 1992). When expressed on the surface of yeast, properly folded CcdB can be detected by binding to FLAG tagged GyrA14 followed by incubations of cells with anti FLAG primary and fluorescently labeled secondary antibody (Sahoo et al, 2015). We selected four PIMs based on their in vitro thermal stability and in vivo activity in E.coli (Adkar et al, 2012; Sahoo et al, 2015). PIM

V18D is completely inactive and highly aggregation prone due to a charged mutation in the core of the protein. V18G and V20G are partially inactive in vitro due to the formation of a cavity in the core of the protein, with V20G showing higher activity in vivo compared to V18G (Adkar et al, 2012).

L36A is the most active amongst the four PIMs, the mutant protein is partially aggregated (Sahoo et

al, 2015). SSSM libraries were constructed by introducing each PIM individually in the background of the single-site saturation mutagenesis library (Sahoo et al, 2015). These SSSM libraries showed variable expression and binding depending on the PIM present in the single mutant library (Supplementary Figure S1). Different populations of SSSM libraries were sorted based on the expression and binding histograms of the libraries (Supplementary Figure S2). Populations were subjected to deep sequencing and the mean fluorescence intensities of binding $\left(\mathrm{MFI}_{\text {seq }}\right.$ (bind)) and expression ( $\mathrm{MFI}_{\text {seq }}\left(\right.$ expr)) were estimated as described (Ahmed et al, 2021). Values of $\mathrm{MFI}_{\text {seq }}$ (bind) and $\mathrm{MFI}_{\text {seq }}$ (expr) were used to predict stabilized mutants. Putative stabilized mutants were identified as those for which either binding or expression is enhanced by at least twenty five percent compared 
123 point mutation in the WT background and measuring the $\mathrm{T}_{\mathrm{m}}$ using thermal shift assay (TSA). In case

124 of the V18D library, we observed that most mutations have low values of normalized MFI $_{\text {seq }}$ (bind) as well as $\mathrm{MFI}_{\text {seq }}$ (expr). This might be because highly destabilizing PIM cannot be rescued by a single suppressor mutation. We observed a low sensitivity and specificity but reasonable accuracy of prediction for stabilized mutants when predictions were made based on either $\mathrm{MFI}_{\text {seq }}$ (bind) or $\mathrm{MFI}_{\text {seq }}$ (expr) (Supplementary Figure S3, Table 1) for this library. While the overall correlation between $\mathrm{MFI}_{\text {seq }}$ values of expression or binding with stability of the mutants was poor (Figure 2A, 2E), nevertheless the best binders showed significant stabilization. Since the results were not as promising as the other libraries, the V18D library was not included in subsequent analyses. A similar analysis was performed for the other three libraries and we found a high sensitivity of prediction for stabilized mutants for these libraries when $\mathrm{MFI}_{\text {seq }}$ (bind) was used as the criterion (Table 1). SSSM libraries with V20G or V18G as PIM displayed the highest sensitivity of prediction of stabilizing mutations (Figure 3A, Supplementary Figure S4A). We found some false positives for the L36A SSSM library (Supplementary Figure S5A), which had similar expression and binding compared to the single-site saturation mutant library of WT CcdB (Supplementary Figure S1). When stabilized mutant predictions were made based on the $\mathrm{MFI}_{\text {seq }}$ (expr), we observed lower sensitivity compared to the predictions based on MFI $_{\text {seq }}$ (bind) (Figure 3B, Supplementary Figure S4B, S5B, Table 1) for all libraries. We also found a good correlation between the $\mathrm{MFI}_{\text {seq }}$ (bind) and the stability of stabilized and marginally destabilized $\left(0>\Delta T_{m}>-5\right)$ mutants (Figure 2).

We hypothesized that the second mutation which alleviates the destabilizing effect of a PIM may act as a global suppressor and can therefore alleviate the destabilizing effects of other PIMs. To confirm this, common suppressors were shortlisted which were present in different PIM libraries (Figure 4).

True global suppressors were identified as those which alleviated the destabilizing effect of at least two PIMs. Using this criterion to identify stabilized mutants we found a sensitivity of 1 for the 
phenotypes, which indicates that the WT is not the most preferred in terms of stability, this information can also be used as a criterion to find the stabilizing mutation. Interestingly none of the suppressors were found at the residues directly involved in GyrA binding. In any case, we have recently shown that such active-site residues can be identified based on the pattern of $\mathrm{MFI}_{\text {seq }}$ (bind) and $\mathrm{MFI}_{\text {seq }}($ expr) 152 in single-site saturation mutagenesis libraries and removed from the set of putative global suppressors 153 (Ahmed et al, 2021).

\section{Additive effect of stabilizing mutations}

While single mutations usually do not enhance the stability of proteins to a great extent, individual stabilizing mutations can be combined resulting in multi-mutants with enhanced stability. We therefore constructed multiple double and triple mutants and measured their thermal stabilities using a thermal shift assay. All the double, triple and multi-mutants showed a higher $\mathrm{T}_{\mathrm{m}}$ than the WT (Figure 5A). We also observed a good correlation $(\mathrm{r}=0.98)$ between $\Delta \mathrm{T}_{\mathrm{m}}$ of double/triple mutants with the sum of $\Delta \mathrm{T}_{\mathrm{m}}$

of individual mutants (Figure 5B). When more than 7 mutants were combined such additive effects

were not observed. While combining 7 or more stabilizing mutations, we did not consider any centroidcentroid distance cut-off, which indicates possible epistatic interactions between residues in close proximity.

\section{Thermal aggregation analysis of stabilized mutants}

To ascertain the ability of mutations to prevent loss of function from transient high temperature exposure, $\mathrm{CcdB}$ mutants were incubated at elevated temperatures for 1 hour, this can result in unfolding of CcdB and subsequent irreversible aggregation (Bajaj et al, 2004). The fraction of active protein remaining after incubation was assessed by its ability to bind GyrA at room temperature using SPR. In the case of WT, we did not observe a large decrease in the fraction of active protein except when incubated at temperatures at and above $60{ }^{\circ} \mathrm{C}$ (Figure $6 \mathrm{~A}$ ). After incubation at $80{ }^{\circ} \mathrm{C}$, the protein was completely denatured, and did not show any binding with Gyrase. In contrast, one stabilized single 
mutant R10G and three multi-site mutants retained significant activity after incubation at $80{ }^{\circ} \mathrm{C}$ for one

173 hour (Figure 6B). Six mutants showed a higher fraction of active protein than WT after heating at 60

$174{ }^{\circ} \mathrm{C}$ (Figure 6C). Three single mutants and two double mutants showed no reduction in active fraction 175 of protein. Surprisingly, the triple mutant, (R10G/A37V/R86H) which showed very high thermal 176 stability, aggregated completely at $80{ }^{\circ} \mathrm{C}$ showing that thermal stability and thermal tolerance need not 177 always be correlated.

\section{Sorting of multiple libraries simultaneously}

179 When the suppressor mutations were identified using both enhanced binding to ligand relative to the corresponding PIM, as well as alleviating the destabilizing effect of at least two PIMs as the criteria, predictions were highly specific. Hence, to find a larger number of stabilizing mutants, it is desirable to screen multiple PIM libraries. However, screening multiple individual libraries is laborious. In most FACS based library screens, individual libraries are sorted for multiple rounds to enrich for the best binders. If this approach is applied to pooled libraries with multiple PIMs, the most stable PIM will dominate, resulting in the enrichment of mutants only from this library. To confirm this, we therefore sorted the pooled library as explained (Supplementary Figure S6A) and we found that mutants with the highest binding were largely from the L36A library as this PIM has the weakest effect on binding (Supplementary Figure S6B). To overcome this problem, we instead sorted multiple populations based on binding of the pooled library (Supplementary Figure S6C) and compared the relative binding of putative (PIM, suppressor) pairs with those of individual PIMs. We found a good correlation between the $\mathrm{MFI}_{\text {seq }}$ (bind) of mutants from the pooled library, with those from individually analysed libraries (Supplementary Figure S6D), with an increase in the correlation as the read cut-off increased. This suggests that single round sorting of YSD pooled SSSM libraries can rapidly identify stabilized mutations. 


\section{Discussion}

197 Directed evolution has drastically reduced the time required to design proteins with new activities.

198 Directed evolution is performed most often in conjunction with display techniques like phage and yeast surface display, which involve selection of better binders to a target of interest from large libraries.

200 While this approach readily selects for high affinity binders, selecting for stable proteins is more difficult. Phage display libraries have very high diversity (Chao et al, 2004). However, in phage display experiments, there is less control over the selection of populations during enrichment and effects of post-translational modification including glycosylation cannot be studied. Yeast display enables the selection of populations during enrichment using FACS (Shusta et al, 2008). Previously, to find stabilized variants of proteins with high intrinsic stability, YSD libraries were subjected to thermal stress, to enrich for more stable variants followed by sorting to identify variants which retained binding to a conformation specific ligand (Pavoor et al, 2012). While this is potentially useful, yeast cells cannot replicate after high temperature exposure, and hence the method requires repeated rounds of plasmid isolation, PCR amplification and retransformation in yeast cells after each round of in such cases will be difficult. Nevertheless, it would be interesting to couple this methodology to the single round sorting and analysis described in the present study. In a recent report, expression level was used to identify a few stabilized mutants of SARS CoV-2 RBD (Starr et al, 2020). In the present study, we did not see a good correlation between expression level of individual mutants and thermal stability of stabilized mutants. Instead, we observed that $\mathrm{MFI}_{\text {seq }}$ (bind) is a better predictor of protein stability than $\mathrm{MFI}_{\text {seq }}$ (expr). Previously, we found that for stable proteins it is difficult to isolate stabilizing mutants using yeast surface display, as the surface expression and binding of all the mutants above a threshold stability are often similar (Ahmed et al, 2021). To overcome this problem, we introduced a PIM in the SSM library of CcdB which reduced binding of all the mutants present in the 
hypothesized that if a stabilizing mutation alleviates the destabilizing effect of at least two PIMs, it is in the SSM library.

224 For libraries with the V18G, V20G and L36A PIMs used in the study, a high specificity for the prediction was observed. These PIM libraries also showed a good correlation between protein stability and MFIseq (bind) (Figure 2). The identified mutants also showed additive stabilization when double or triple mutants were combined.

We also found that if a greater number of PIM libraries are screened, it enhances the prediction of stabilized mutants. To decrease the time and effort involved in screening multiple libraries, we pooled multiple libraries. The pooled library showed similar reconstructed MFIs of mutants to those obtained from individually analysed libraries, validating the approach used. Importantly, the size of libraries employed here is relatively small, identical to single-site saturation mutagenesis libraries, with a total size of $32 \mathrm{~N}$, where $\mathrm{N}$ is the length of the protein sequence. It can thus easily be extended to other library technologies including lentiviral and transposon libraries, phage and mammalian display.

The methodology has the following limitations. The methodology requires a conformation specific ligand to differentiate between the destabilized PIM and stabilized PIM-suppressor pair. For some proteins hyper glycosylation on the yeast cell surface may interfere with the binding between yeast surface displayed protein and its ligand. As an alternative to ligand binding, protease resistance can also be potentially used to select for stabilized mutants (Rocklin et al, 2017; Golinski et al, 2021). However, the aggregation and unfolding on the yeast cell surface may limit the cleavage of such proteins. Use of a protease assay to screen for stabilized mutants, assumes that protease cleavage occurs primarily in the unfolded state (Park \& Marqusee, 2005). While this is true for small well folded proteins, for larger proteins, initial sites of cleavage may occur at surface loops, complicating 
245 to identify stabilizing mutations in any protein of interest for which a surface display based binding

246 screen is available.

\section{Materials and methods}

\section{Bacterial strains, yeast strains and plasmids}

249 E.coli strain Top10Gyrase has a mutation in the gyrA gene which prevent CcdB toxicity. The EBY100 250 strain of Saccharomyces cerevisiae has TRP1 mutation which make it auxotrophic for tryptophan and 251 transformants can be selected on minimal media. WT and mutant $c c d B$ genes were coned in pBAD24 252 plasmid for controllable expression in E.coli. pPNLS shuttle vector was used to display ccdB mutants 253 for yeast cell surface expression.

\section{Purification of WT and mutant CcdB proteins}

255 CcdB WT and mutant proteins were purified as described (Chattopadhyay \& Varadarajan, 2019), 256 briefly, overnight grown culture was diluted 100 folds in $300 \mathrm{ml} \mathrm{LB}$ media containing ampicillin $257(100 \mu \mathrm{g} / \mathrm{ml})$. The cells were grown and induce at an $\mathrm{OD}_{600} \sim 0.5$ for 3 hours at $37{ }^{\circ} \mathrm{C}$. The cells were 258 harvested after induction and lysed using sonication. The soluble fraction was separated using 259 centrifugation and incubated with Affigel-15 coupled to CcdA peptide (residue 45-72) for 2 hours at $2604{ }^{\circ} \mathrm{C}$. The unbound fraction was removed, and beads were washed with bicarbonate buffer $(50 \mathrm{mM}$ $261 \mathrm{NaHCO} 3$ and $500 \mathrm{mM} \mathrm{NaCl})$. The proteins were eluted with glycine (200 mM, pH 2.5) and collected 262 in tubes containing an equal volume of HEPES buffer (400 mM, pH 8)

\section{Second site Saturation Suppressor Mutagenesis (SSSM) library generation}

264 A ccdB site saturation mutagenesis (SSM) library was generated through an inverse PCR based approach (Jain \& Varadarajan, 2014). The second site suppressor mutant library for CcdB was generated by introducing PIMs in the SSM library as described (Sahoo et al, 2015). 
Yeast surface expression and sorting of SSSM library

Yeast surface display and flowcytometric analysis were performed as explained earlier (Ahmed et al,

2021). Briefly, Saccharomyces cerevisiae EBY100 cells containing WT ccdB or mutant plasmids were

grown in SDCAA media for 16 hours and induced in SGCAA media for an additional 16 hours at 30

${ }^{\circ} \mathrm{C}$. Ten million cells were taken for FACS sample preparation. The Second site Suppressor mutant

273 library of CcdB was sorted based on 1D sorting of surface expression and binding to1000 nM GyrA14.

274 The estimated $\mathrm{K}_{\mathrm{d}}$ for WT CcdB to GyrA14 is $4 \mathrm{nM}$. A higher CcdB concentration was employed to 275 ensure that even destabilized mutants where only a small fraction was properly folded, showed 276 detectable binding to $\mathrm{CcdB}$. Surface expression was quantitated by fluorescent antibody binding to 277 HA tagged CcdB. Binding to GyrA14 was detected by anti FLAG antibody as described previously 278 (Sahoo et al, 2015). The sorting of CcdB libraries was performed using a BD Aria III cell sorter. In 279 the case of simultaneous sorting of multiple SSSM libraries, each library samples were prepared 280 separately as explained above and pooled before sorting.

\section{Sample preparation for deep sequencing}

282 Deep sequencing samples were prepared as explained earlier (Ahmed et al, 2021). Briefly, sorted cells were grown on SDCAA agar plates, colonies were scraped, and pooled plasmids were extracted. The ccdB gene was PCR amplified using the primers having multiplex identifier (MID) sequence at the 5'end, that bind upstream and downstream of the ccdB gene. The DNA was PCR amplified for 15 cycles, equal amounts of DNA from each sorted population were pooled, gel extracted, and the library was generated using TruSeq ${ }^{\mathrm{TM}}$ DNA PCR-Free kit from Illumina. The sequencing was done on an

Illumina HiSeq 2500 250PE platform at Macrogen, South Korea.

\section{Analysis of deep sequencing data}

Deep sequencing data for the ccdB mutants were processed as described (Ahmed et al, 2021). Briefly, 
$a l, 2014)$. Following assembly, reads were filtered to eliminate those that do not contain the relevant

293 MID and/or primers along with the reads having mismatched MID's. Only those reads which have

294 bases with Phred score $\geq 20$ are retained. Reads which pass the assembling and filtering step were

295 binned according to the respective MIDs. Binned reads were aligned with the wild type ccdB sequence

296 using the Water v6.4.0.0 program (Smith \& Waterman, 1981) and reformatted. Finally the reads were 297 classified based on insertions, deletions and substitutions (single, double, etc mutants).

\section{MFI reconstruction from deep sequencing data}

The MFI of each mutant was reconstructed as described (Ahmed et al, 2021). Briefly, reads of each mutant were normalized across different bins (Equation 1). The fraction of each mutant $(\mathrm{X} i)$ distributed across each bin was calculated (Equation 2). The reconstructed MFI ( $\left.\mathrm{MFI}_{\text {seq }}\right)$ of individual mutant was calculated by the summation of the product, obtained upon multiplying the fraction $(\mathrm{X} i)$ of that mutant in bin ( $i$ ) with the MFI of the corresponding bin obtained from the FACS experiment (Fi), across the various bins populated by that mutant (Equation 3). The normalized MFI of each mutant was calculated from the reconstructed MFI of each mutant (Equation 4).

Normalized read of mutant in bin $i(\mathrm{~N} i)=\frac{\text { No.of mutant } i \text { in bin } i}{\sum \text { Reads } \text { in bin } i}$ ... Equation 1

Fraction of mutants in each gate $(\mathrm{X} i)=\frac{N i}{\sum_{1}^{n} N i}$ ... Equation 2

Normalized MFI $=\frac{\text { Reconstructed MFI of mutant } i}{\text { Reconstructed MFI of mutant } W T}$ ... Equation 4

Stabilized mutants were classified as those which showed at least $25 \%$ enhanced binding or expression when present as PIM-suppressor pair compared to the PIM alone. 


\section{Protein thermal stability measurement.}

This was carried out as described (Chattopadhyay \& Varadarajan, 2019). Briefly, a solution of total volume $20 \mu \mathrm{L}$ containing $10 \mu \mathrm{M}$ of the purified $\mathrm{CcdB}$ protein and 2.5x Sypro orange dye in buffer (200 mM HEPES, $100 \mathrm{mM}$ glycine), $\mathrm{pH} 7.5$ was heated from $15{ }^{\circ} \mathrm{C}$ to $90{ }^{\circ} \mathrm{C}$ with $0.5{ }^{\circ} \mathrm{C}$ increment every 30 seconds on an iCycle iQ5 Real Time Detection System (Bio-Rad, Hercules, CA). The normalized fluorescence data was plotted against temperature (Niesen et al, 2007).

\section{Thermal aggregation studies of CcdB mutants.}

321 CcdB mutants and WT proteins ( $500 \mathrm{nM}, 200 \mu \mathrm{L}$ for each of the proteins) were subjected to incubation 322 at four different temperatures, 20, 40, 60 and $80{ }^{\circ} \mathrm{C}$ on an iCycle iQ5 Real Time Detection System 323 (Bio-Rad, Hercules, CA). The temperature was gradually increased to the desired temperature at a rate 324 of $3{ }^{\circ} \mathrm{C} / \mathrm{min}$ and samples were kept at the desired temperature for 1 hour. The heated protein was then 325 cooled down to $4{ }^{\circ} \mathrm{C}$ at the rate of $3{ }^{\circ} \mathrm{C} / \mathrm{min}$. The aggregated protein was removed using centrifugation 326 at $18000 \mathrm{~g}$. The fraction of active protein remaining was measured by binding to GyrA14 on a Biacore 3272000 SPR platform. The percentage of active protein at different temperatures was calculated using 328 the following equation.

$$
\% \text { Active }=\frac{\text { Binding of CcdB after incubation at temperature } T(R U)}{\text { Binding of CcdB after incubation at } 20{ }^{\circ} \mathrm{C}(R U)} * 100
$$




\section{Statistical Analysis}

All the data were plotted using the GraphPad Prism software 9.0.0. The correlation coefficients between deep sequencing replicates were estimated using the GraphPad Prism software 9.0.

\section{Data Availability}

All the data are in the manuscript.

Competing Interest Statement: The authors claim no conflict of interest.

\section{Author contribution}

R.V. and S.A. designed the experiments. S.A. performed all the experiments. R.V. and S.A. analyzed all the data. K.M. wrote the software and carried out the processing of the deep sequencing data. S.A. analyzed the deep sequencing data of CcdB. R.V. and S.A. wrote most of the manuscript.

\section{Acknowledgements}

This work was funded in part by a grant to RV from the Department of Science and Technology, grant number-EMR/2017/004054, DT.15/12/2018), Government of India and Department of Biotechnology, grant no. BT/COE/34/SP15219/2015 DT. 20/11/2015, Ministry of Science and Technology, Government of India. We also acknowledge funding for infrastructural support from the following programs of the Government of India: DST FIST, UGC Centre for Advanced study, Ministry of Human Resource Development (MHRD), and the DBT IISc Partnership Program. The funders had no role in study design, data collection and interpretation, or the decision to submit the work for publication. S.A. acknowledges Council of Scientific \& Industrial Research for his fellowship (SPM07/079(0218)/2015-EMR-I). K.M. is thankful to Department of Science and Technology (DST) Science and Engineering Research Board for financial support, sanction order no: PDF/2017/002641. Aparna Ashok is duly acknowledged for FACS. Nonavinakere Seetharam Srilatha is duly acknowledged for the SPR experiments. 


\section{References}

Adkar B V., Tripathi A, Sahoo A, Bajaj K, Goswami D, Chakrabarti P, Swarnkar MK, Gokhale RS \&

Varadarajan R (2012) Protein model discrimination using mutational sensitivity derived from deep

20:

$371-381$

Available

at:

https://www.sciencedirect.com/science/article/pii/S0969212612000068 [Accessed June 6, 2019]

Ahmed S, Manjunath K \& Varadarajan R (2021) Prediction of residue-specific contributions to

binding and thermal stability using yeast surface display. bioRxiv: 2021.05.31.446445 Available at: http://biorxiv.org/content/early/2021/06/01/2021.05.31.446445.abstract

Araya CL, Fowler DM, Chen W, Muniez I, Kelly JW \& Fields S (2012) A fundamental protein property, thermodynamic stability, revealed solely from large-scale measurements of protein function.

Proc.

Natl.

Acad.

Sci.

109:

$16858-16863$

Available

at:

http://www.pnas.org/cgi/doi/10.1073/pnas.1209751109 [Accessed July 11, 2019]

Bajaj K, Chakshusmathi G, Bachhawat-Sikder K, Surolia A \& Varadarajan R (2004) Thermodynamic characterization of monomeric and dimeric forms of $\mathrm{CcdB}$ (controller of cell division or death B

protein). Biochem. J. 380: 409-17 Available at: http://www.ncbi.nlm.nih.gov/pubmed/14763902

374 [Accessed July 27, 2019]

Bernard P \& Couturier M (1992) Cell killing by the F plasmid CcdB protein involves poisoning of

DNA-topoisomerase II complexes. J. Mol. Biol. 226: 735-745 Available at:

377 https://www.sciencedirect.com/science/article/pii/002228369290629X [Accessed June 8, 2019]

378 Blacklow SC, Liu KD \& Knowles JR (1991) Stepwise improvements in catalytic effectiveness: 379 independence and interdependence in combinations of point mutations of a sluggish triosephosphate isomerase. Biochemistry 30: 8470-6 Available at: http://pubs.acs.org/doi/abs/10.1021/bi00098a026 [Accessed July 26, 2019] 
Boder ET \& Wittrup KD (1997) Yeast surface display for screening combinatorial polypeptide

libraries. Nat. Biotechnol. 15: 553-7 Available at: http://www.ncbi.nlm.nih.gov/pubmed/9181578

[Accessed July 11, 2019]

Brachmann RK, Yu K, Eby Y, Pavletich NP \& Boeke JD (1998) Genetic selection of intragenic

suppressor mutations that reverse the effect of common p53 cancer mutations. EMBO J. 17: 1847-59

Available at: http://emboj.embopress.org/cgi/doi/10.1093/emboj/17.7.1847 [Accessed July 27, 2019]

Chao G, Cochran JR \& Wittrup KD (2004) Fine epitope mapping of anti-epidermal growth factor

receptor antibodies through random mutagenesis and yeast surface display. J. Mol. Biol. 342: 539-550

Chattopadhyay G \& Varadarajan R (2019) Facile measurement of protein stability and folding kinetics

using a nano differential scanning fluorimeter. Protein Sci. 28: 1127-1134 Available at:

https://onlinelibrary.wiley.com/doi/abs/10.1002/pro.3622 [Accessed July 26, 2019]

393 Esteban O \& Zhao H (2004) Directed evolution of soluble single-chain human class II MHC

molecules.

J. Mol.

Biol.

340:

$81-95$

Available

at:

https://www.sciencedirect.com/science/article/pii/S0022283604005017?via\%3Dihub\#BIB25

[Accessed June 6, 2019]

Foit L, Morgan GJ, Kern MJ, Steimer LR, von Hacht AA, Titchmarsh J, Warriner SL, Radford SE \&

Bardwell JCA (2009) Optimizing Protein Stability In Vivo. Mol. Cell 36: 861-871 Available at:

http://www.ncbi.nlm.nih.gov/pubmed/20005848 [Accessed July 27, 2019]

Golinski AW, Mischler KM, Laxminarayan S, Neurock NL, Fossing M, Pichman H, Martiniani S \&

Hackel BJ (2021) High-throughput developability assays enable library-scale identification of producible protein scaffold variants. Proc. Natl. Acad. Sci. 118: Available at: 
sequences. Proc. Natl. Acad. Sci. U. S. A. 99: 6619-6624 Available at:

407 Hermes JD, Blacklow SC \& Knowles JR (1990) Searching sequence space by definably random mutagenesis: improving the catalytic potency of an enzyme. Proc. Natl. Acad. Sci. U. S. A. 87: 696700 Available at: http://www.pnas.org/cgi/doi/10.1073/pnas.87.2.696 [Accessed July 22, 2019]

Jain PC \& Varadarajan R (2014) A rapid, efficient, and economical inverse polymerase chain reaction-

411 based method for generating a site saturation mutant library. Anal. Biochem. 449: 90-8 Available at: 412 http://www.ncbi.nlm.nih.gov/pubmed/24333246

413 Lehtovaara PM, Koivula AK, Bamford J \& Knowles JK (1988) A new method for random mutagenesis 414 of complete genes: enzymatic generation of mutant libraries in vitro. Protein Eng. 2: 63-8 Available 415 at: http://www.ncbi.nlm.nih.gov/pubmed/3151018 [Accessed July 22, 2019]

416 Maxwell KL, Mittermaier AK, Forman-Kay JD \& Davidson AR (1999) A simple in vivo assay for 417 increased protein solubility. Protein Sci. 8: 1908-1911 Available at: 418 http://www.ncbi.nlm.nih.gov/pubmed/10493593 [Accessed July 27, 2019]

419 Mimmi S, Maisano D, Quinto I \& Iaccino E (2019) Phage Display: An Overview in Context to Drug 420 Discovery. Trends Pharmacol. Sci. 40: 87-91 Available at: 421 https://linkinghub.elsevier.com/retrieve/pii/S016561471830230X [Accessed July 11, 2019]

422 Niesen FH, Berglund H \& Vedadi M (2007) The use of differential scanning fluorimetry to detect 423 ligand interactions that promote protein stability. Nat. Protoc. 2: 2212-2221 Available at: 424 http://www.ncbi.nlm.nih.gov/pubmed/17853878 [Accessed June 6, 2019]

425 Nikolova P V. (2000) Mechanism of rescue of common p53 cancer mutations by second-site 426 suppressor mutations. EMBO J. 19: 370-378

427 Park C \& Marqusee S (2005) Pulse proteolysis: A simple method for quantitative determination of 
428 protein stability and ligand binding. Nat. Methods 2: 207-212 Available at:

429 https://pubmed.ncbi.nlm.nih.gov/15782190/ [Accessed August 5, 2021]

430 Park S, Xu Y, Stowell XF, Gai F, Saven JG \& Boder ET (2006) Limitations of yeast surface display 431 in engineering proteins of high thermostability. Protein Eng. Des. Sel. 19: 211-217 Available at: 432 http://academic.oup.com/peds/article/19/5/211/1556411/Limitations-of-yeast-surface-display-in 433 [Accessed June 6, 2019]

434 Pavoor T V., Wheasler JA, Kamat V \& Shusta E V. (2012) An enhanced approach for engineering 435 thermally stable proteins using yeast display. Protein Eng. Des. Sel. 25: 625-630 Available at: /pmc/articles/PMC3449400/?report=abstract [Accessed November 12, 2020]

437 Piatesi A, Howland SW, Rakestraw JA, Renner C, Robson N, Cebon J, Maraskovsky E, Ritter G, Old 438 L \& Wittrup KD (2006) Directed evolution for improved secretion of cancer-testis antigen NY-ESO4391 from yeast. Protein Expr. Purif. 48: 232-242 Available at: 440 https://linkinghub.elsevier.com/retrieve/pii/S1046592806000337 [Accessed June 6, 2019]

441 Rocklin GJ, Chidyausiku TM, Goreshnik I, Ford A, Houliston S, Lemak A, Carter L, Ravichandran 442 R, Mulligan VK, Chevalier A, Arrowsmith CH \& Baker D (2017) Global analysis of protein folding 443 using massively parallel design, synthesis, and testing. Science 357: 168-175 Available at: 444 http://www.ncbi.nlm.nih.gov/pubmed/28706065

445 Sahoo A, Khare S, Devanarayanan S, Jain PC \& Varadarajan R (2015) Residue proximity information 446 and protein model discrimination using saturation-suppressor mutagenesis. Elife 4: e09532 Available 447 at: https://elifesciences.org/articles/09532 [Accessed July 18, 2019]

448 Schwimmer LJ, Huang B, Giang H, Cotter RL, Chemla-Vogel DS, Dy F V., Tam EM, Zhang F, Toy 449 P, Bohmann DJ, Watson SR, Beaber JW, Reddy N, Kuan HF, Bedinger DH \& Rondon IJ (2013) 450 Discovery of diverse and functional antibodies from large human repertoire antibody libraries. $J$. 451 Immunol. Methods 391: 60-71 
452 Shusta E, Pepper L, Cho Y \& Boder E (2008) A Decade of Yeast Surface Display Technology: Where Are We Now? Comb. Chem. High Throughput Screen. 11: 127-134

454 Shusta E V., Kieke MC, Parke E, Kranz DM \& Wittrup KD (1999) Yeast polypeptide fusion surface 455 display levels predict thermal stability and soluble secretion efficiency. J. Mol. Biol. 292: 949-956 456 Available at: http://www.ncbi.nlm.nih.gov/pubmed/10512694

Smith TF \& Waterman MS (1981) Identification of common molecular subsequences. J. Mol. Biol. 147: 195-197 Available at: http://www.ncbi.nlm.nih.gov/pubmed/7265238 [Accessed June 6, 2019]

459 Starr TN, Greaney AJ, Hilton SK, Ellis D, Crawford KHD, Dingens AS, Navarro MJ, Bowen JE, 460 Tortorici MA, Walls AC, King NP, Veesler D \& Bloom JD (2020) Deep Mutational Scanning of 182: $1295-1310 . e 20$

463 Tao H \& Cornish VW (2002) Milestones in directed enzyme evolution. Curr. Opin. Chem. Biol. 6: 464 858-64 Available at: http://www.ncbi.nlm.nih.gov/pubmed/12470742 [Accessed July 28, 2019]

465 Tripathi A, Gupta K, Khare S, Jain PC, Patel S, Kumar P, Pulianmackal AJ, Aghera N \& Varadarajan 466 R (2016) Molecular Determinants of Mutant Phenotypes, Inferred from Saturation Mutagenesis Data. Mol. Biol. Evol. 33: 2960-2975

Turner NJ (2009) Directed evolution drives the next generation of biocatalysts. Nat. Chem. Biol. 5: 567-573 Available at: http://www.ncbi.nlm.nih.gov/pubmed/19620998 [Accessed July 28, 2019]

470 Waldo GS, Standish BM, Berendzen J \& Terwilliger TC (1999) Rapid protein-folding assay using 471 green fluorescent protein. Nat. Biotechnol. 17: 691-695 Available at: 472 http://www.nature.com/articles/nbt0799_691 [Accessed July 25, 2019]

473 Wigley WC, Stidham RD, Smith NM, Hunt JF \& Thomas PJ (2001) Protein solubility and folding 474 monitored in vivo by structural complementation of a genetic marker protein. Nat. Biotechnol. 19: 
475 131-136 Available at: http://www.nature.com/articles/nbt0201_131 [Accessed July 25, 2019]

476 Wu C-H, Liu I-J, Lu R-M \& Wu H-C (2016) Advancement and applications of peptide phage display

477 technology in biomedical science. J. Biomed. Sci. 23: 8 Available at:

478 http://www.ncbi.nlm.nih.gov/pubmed/26786672 [Accessed July 11, 2019]

479 Zhang J, Kobert K, Flouri T \& Stamatakis A (2014) PEAR: A fast and accurate Illumina Paired-End 480 reAd mergeR.

Bioinformatics

30:

$614-620$

Available

at:

481 http://www.ncbi.nlm.nih.gov/pubmed/24142950 [Accessed June 6, 2019] 

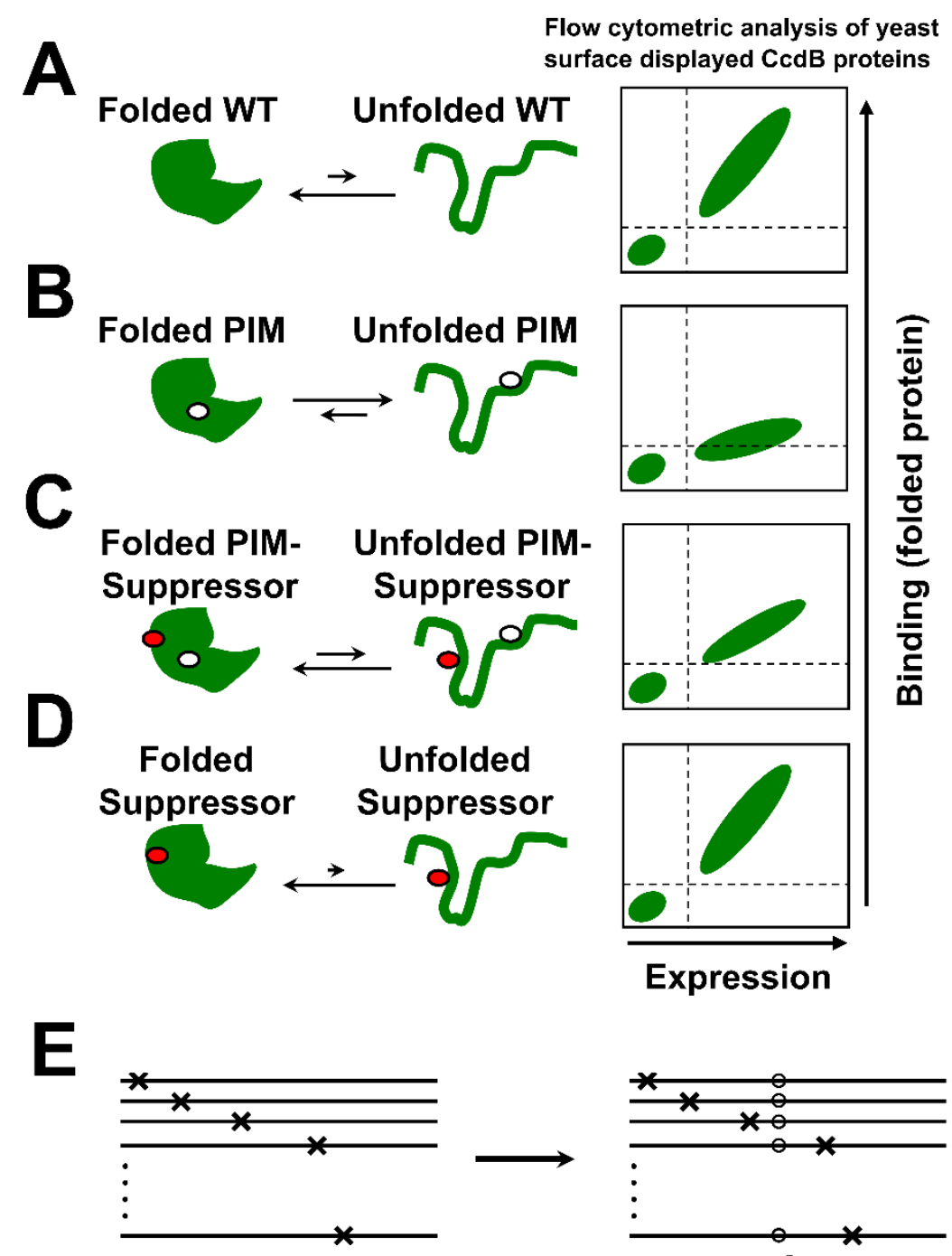

Figure 1: Schematic representation of Second Site Suppressor Mutagenesis (SSSM) methodology. Proteins exist in an equilibrium between folded and unfolded states. (A) WT proteins generally have the equilibrium shifted towards the folded state. Such proteins when expressed on the 501 yeast cell surface show good expression and binding to their cognate ligand (B) Introduction of a 502 Parent Inactive Mutation (PIM) shift the equilibrium towards the unfolded state, and decrease the amount of properly folded protein on the yeast cell surface, leading to decreased ligand binding. (C) 
504 Second-site suppressor mutation, distal from and present in the background of the PIM will reduce the 505 amount of unfolded protein present at equilibrium. Such double mutants have higher expression and 506 binding on the yeast cell surface compared to the PIM alone. (D) Such global/distal suppressor 507 mutations can stabilize multiple PIMs and also stabilize the WT protein, although the expression and 508 binding of suppressor alone on the yeast cell surface is similar to the WT protein. (E) Saturation 509 suppressor libraries are created by introducing a PIM (o) into the background of a single-site (x) 510 saturation mutagenesis library, selecting for suppressors, and reintroducing identified suppressors in 511 the background of the WT gene. 

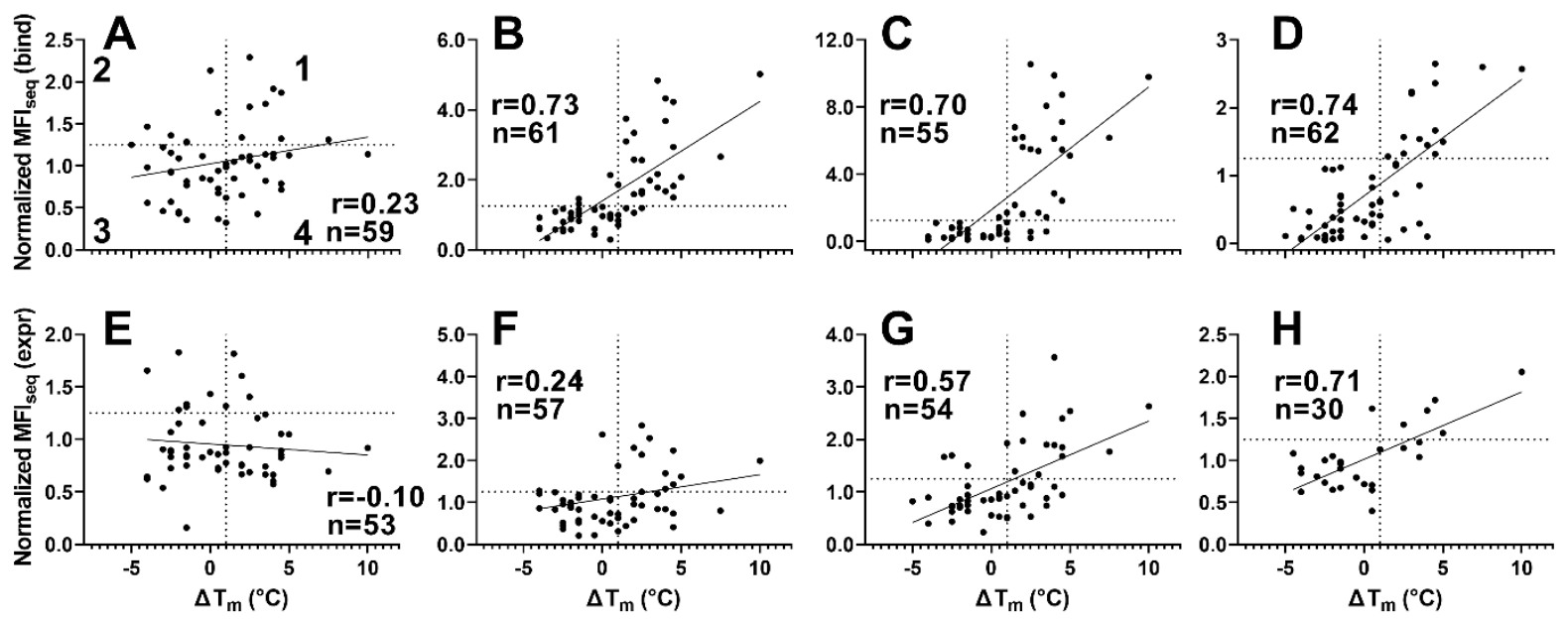

Figure 2: Correlation of CcdB mutant stability ( $\Delta T_{m}$ of single mutant) with normalized MFIseq

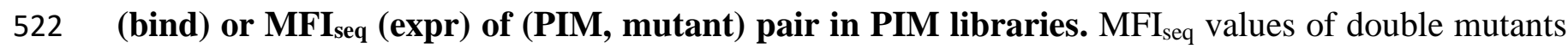

523 were normalized with the $\mathrm{MFI}_{\text {seq }}$ values of their respective PIM. First, second, third and fourth 524 quadrants numbered in (A) represent true positive, false positive, true negative and false negative 525 points respectively. Normalized $\mathrm{MFI}_{\text {seq }}$ (bind) correlation with thermal stability for V18D (A), V18G 526 (B), V20G (C) and L36A (D) libraries. Normalized MFI seq $_{\text {(expr) correlation with thermal stability for }}$ 527 V18D (E), V18G (F), V20G (G) and L36A (H) libraries. Normalized MFI seq $_{\text {(bind) correlates better }}$ 528 than Normalized $\mathrm{MFI}_{\text {seq }}$ (expr) with thermal stability. For V18D while overall correlation is poor, those 529 mutants with highest $\mathrm{MFI}_{\text {seq }}$ (bind) are stabilized. 


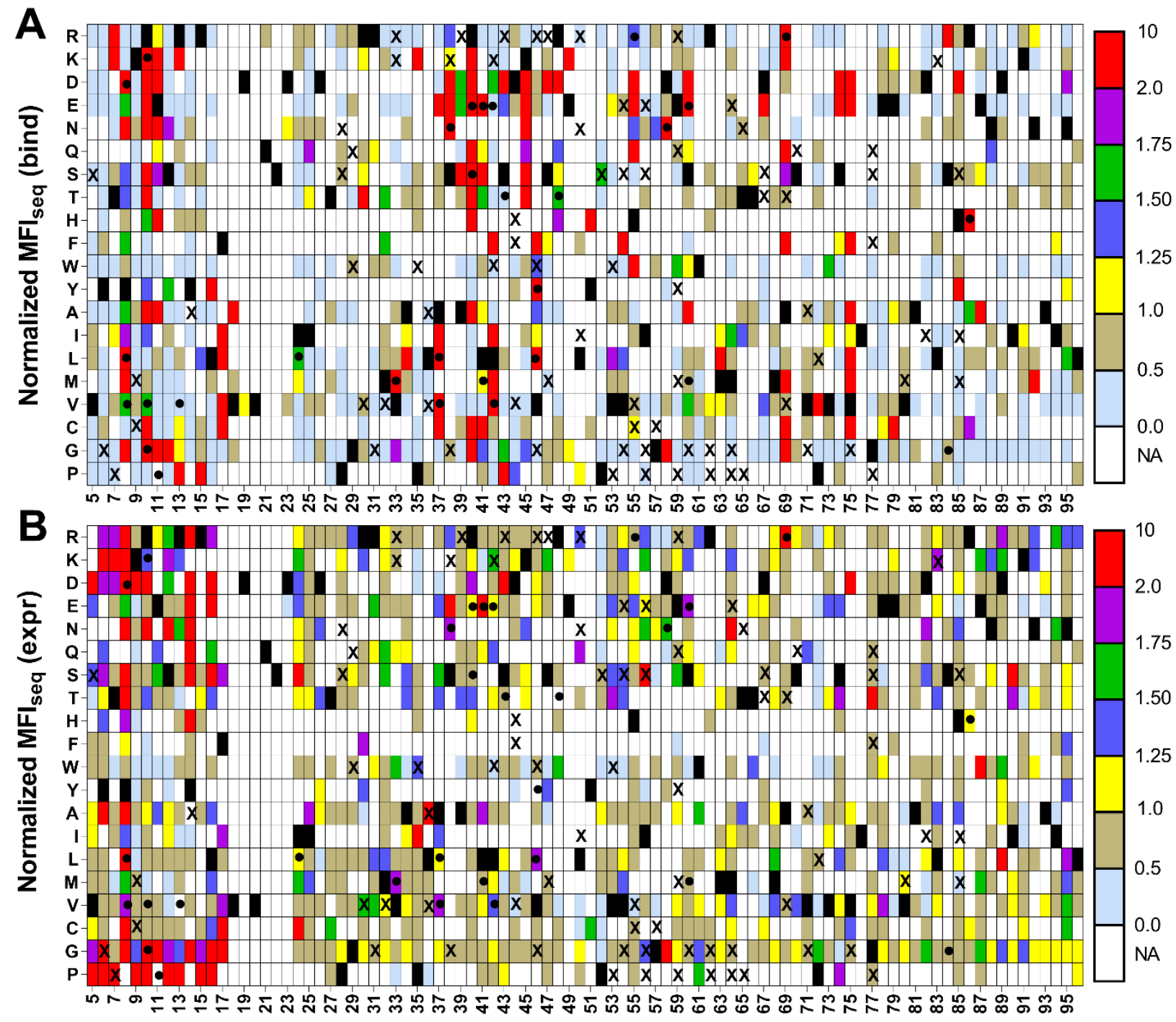

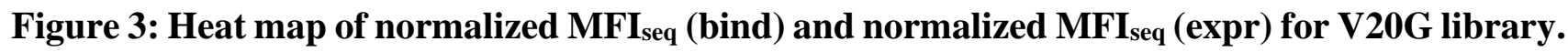

534 Normalized (with respect to $\mathrm{V} 20 \mathrm{G}$ ) values of $\mathrm{MFI}_{\text {seq }}$ (bind) (A) and $\mathrm{MFI}_{\text {seq }}$ (expr) (B) were categorized 535 in different ranges. Mutants with normalized $\mathrm{MFI}_{\text {seq }}$ (bind) or $\mathrm{MFI}_{\text {seq }}$ (expr) greater than 1.25 were 536 categorized as putative stabilized mutants. Black rectangle represents WT residues, mutants where no 537 data are available are indicated with a white rectangle. A subset of mutants were purified and their 538 thermal stability $\left(\mathrm{T}_{\mathrm{m}}\right)$ in vitro was measured. Stable mutants $\left(\Delta \mathrm{T}_{\mathrm{m}}>1\right)$ are indicated with a "•" and 539 destabilized mutants $\left(\Delta \mathrm{T}_{\mathrm{m}}<0\right)$ are indicated with an " $\mathrm{X}$ ". It is clear that $\mathrm{MFI}_{\text {seq }}$ (bind) is superior to $\mathrm{MFI}_{\text {seq }}$ (expr) in identification of stabilized mutants. 


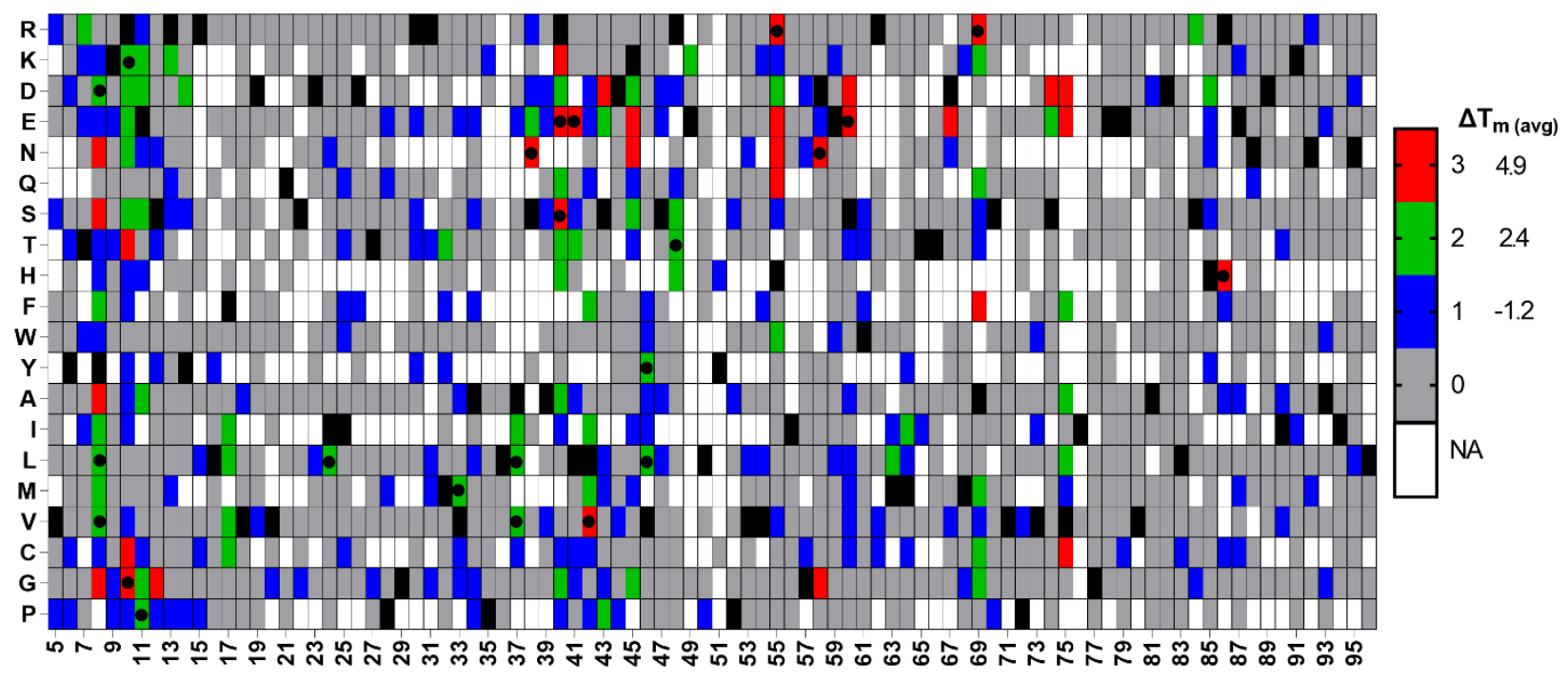

543 Figure 4: Heat map of putative stabilized mutants found in all the three V18G, V20G and L36A

544 libraries based on MFI $_{\text {seq }}$ (bind). Putative suppressor mutants in each library were given a score of 545 one unit. Grey, blue red and green indicate that the mutant acted as a suppressor in zero, one, two and 546 three different libraries. White indicate the mutants where no data are available. 


\section{A}

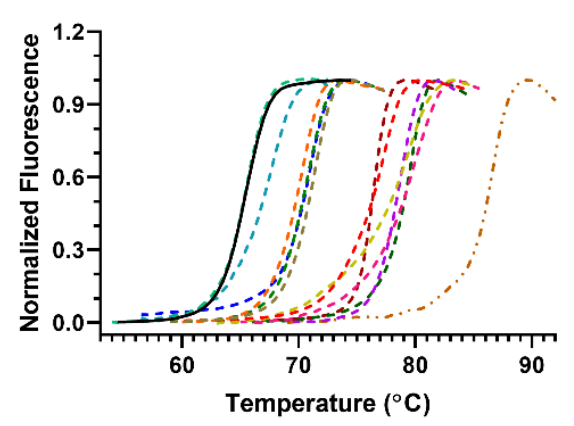

B

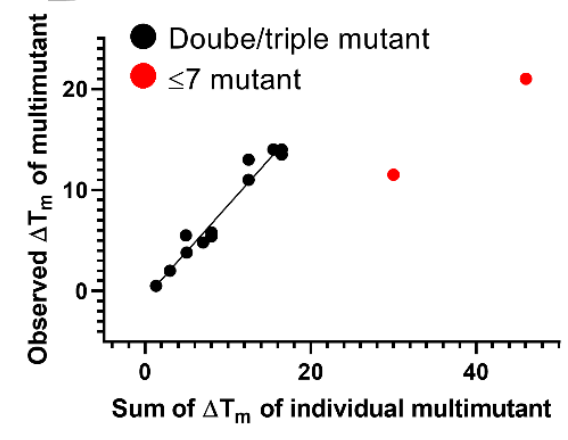

553 Figure 5: Thermal shift assay data for select CcdB double, triple and multi-site mutants. (A)

554 Data for WT and mutants are shown in black and colour respectively. Multi-mutants showed higher

555 thermal stability compared to individual mutants. Mutations present in $7 \mathrm{M}$ are 556 Y8D/R10G/E11P/S12G/A37V/R40S/A69R and 10M are Y8D/R10G/E11P/S12G/A37V/R40S

557 /L42V/V46L/A69R/R86H. (B) The multi-mutants showed an additive effect when two or three 558 mutations were combined. Multi-mutant which contain seven or more mutations did not show 559 completely additive stabilization. 


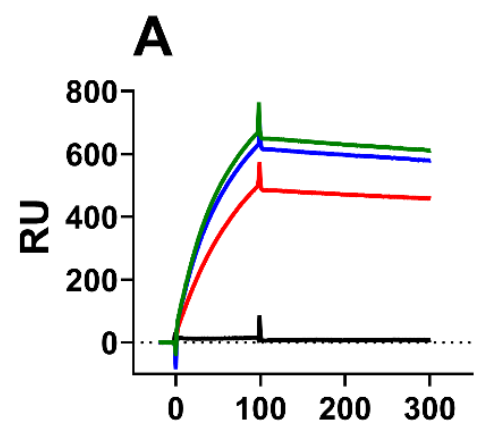

Time

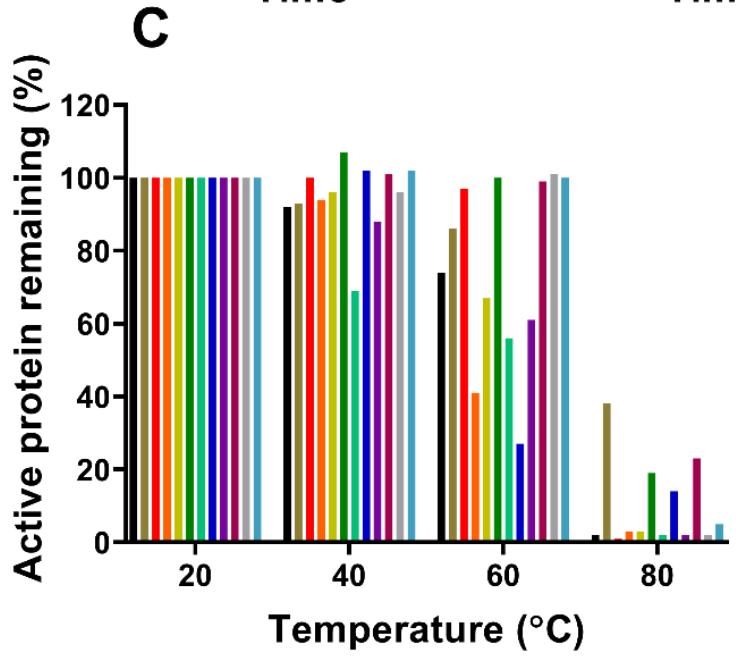

564 Figure 6: Estimation of the fraction of active protein after thermal stress. CcdB WT and mutants were incubated at $20,40,60$ and $80{ }^{\circ} \mathrm{C}$ for one hour. The fraction of active protein was subsequently estimated by assaying binding to GyrA at $25{ }^{\circ} \mathrm{C}$ using SPR. A representative SPR sensorgram for (A)

567 WT CcdB and (B) R10G CcdB showing the relative amount of active protein remaining in the samples

568 after incubation at different temperatures for one hour. (C) Fraction (\%) of active protein after 569 incubation at indicated temperature for 1 hour. 


\section{Tables}

\section{Table 1:}

577 Identification of stabilized mutant solely from normalized $\mathrm{MFI}_{\text {seq }}$ (bind) or $\mathrm{MFI}_{\text {seq }}$ (expr) data. Mutants 578 were predicted to be stabilized if the corresponding $\mathrm{MFI}_{\text {seq }}$ value was greater than 25 percent. CcdB 579 mutant thermal stability data was taken from previous studies (Tripathi et al, 2016; Ahmed et al, 2021) 580 as well as additional mutant stability data measured in this study (Supplementary Table 1).

\begin{tabular}{|l|c|c|c|c|c|c|c|c|}
\hline \multirow{2}{*}{ Parameter } & \multicolumn{2}{|c|}{ V18D library } & \multicolumn{2}{c|}{ V18G library } & \multicolumn{2}{c|}{ V20G library } & \multicolumn{2}{c|}{ L36A library } \\
\cline { 2 - 9 } & Bind & Expr & Bind & Expr & Bind & Expr & Bind & Expr \\
\hline Sensitivity $^{\mathbf{a}}$ & 0.27 & 0.15 & 0.80 & 0.44 & 0.79 & 0.54 & 0.63 & 0.56 \\
\hline Specificity $^{\mathbf{a}}$ & 0.73 & 0.88 & 0.89 & 0.79 & 0.98 & 0.78 & 1.00 & 0.91 \\
\hline Accuracy $^{\mathbf{a}}$ & 0.57 & 0.63 & 0.86 & 0.67 & 0.90 & 0.70 & 0.89 & 0.84 \\
\hline
\end{tabular}

${ }^{\text {a }}$ The sensitivity, specificity and accuracy were calculated using the following formulae, where TP, TN,

FP and FN correspond to true positive $\left(\Delta \mathrm{T}_{\mathrm{m}}\right.$ (predicted $)>0, \Delta \mathrm{T}_{\mathrm{m}}$ (observed) $\left.>0\right)$, true negative $\left(\Delta \mathrm{T}_{\mathrm{m}}\right.$

(predicted) $<0, \Delta \mathrm{T}_{\mathrm{m}}$ (observed) $\left.<0\right)$, false positive $\left(\Delta \mathrm{T}_{\mathrm{m}}\right.$ (predicted) $>0, \Delta \mathrm{T}_{\mathrm{m}}$ (observed) $\left.<0\right)$ and

$586 \quad$ Sensitivity $=\frac{T P}{T P+F N}$

$587 \quad$ Specificity $=\frac{T N}{T N+F P}$ 


\section{Supplementary Information}
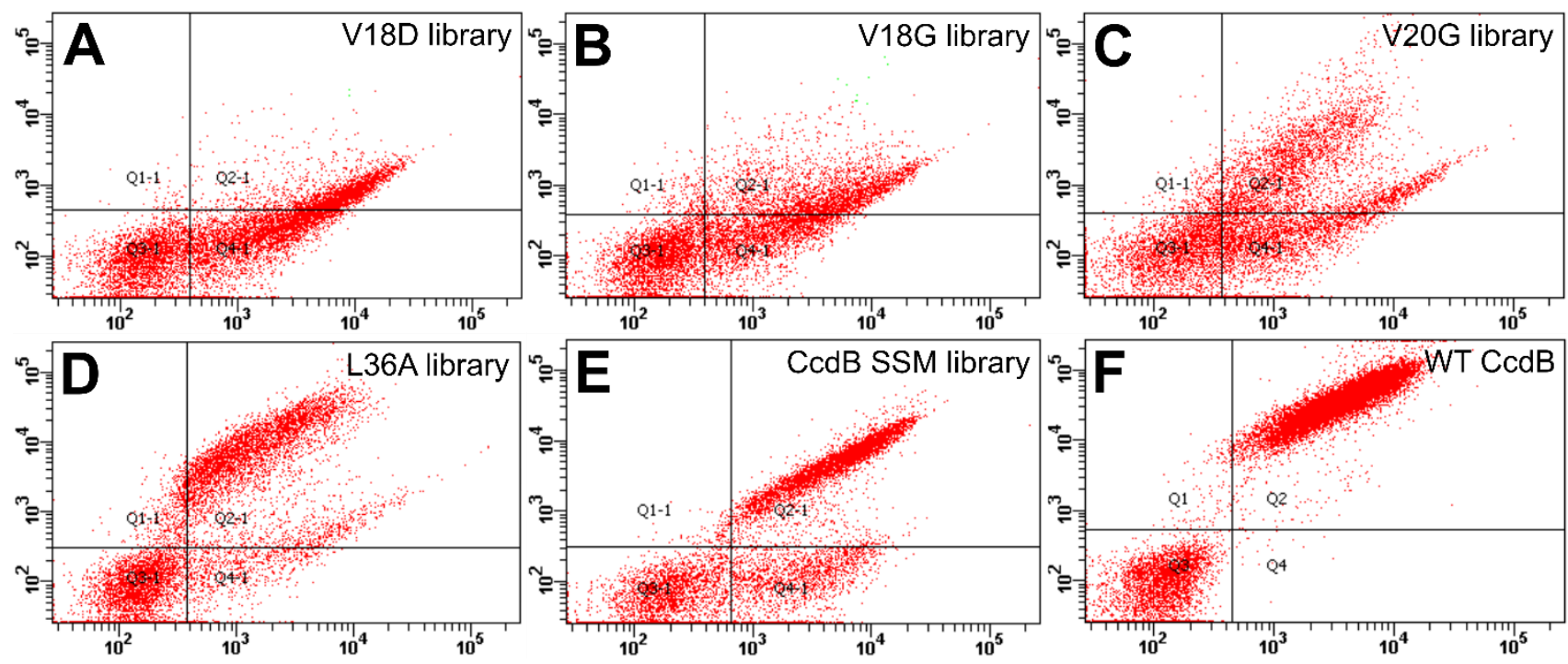

Supplementary Figure S1: FACS double plot showing the expression and binding to GyrA14 of

CcdB mutant libraries in the background of different PIMs. FACS double plot of CcdB library

containing PIMs (A) V18D, (B) V18G, (C) V20G and (D) L36A. Double plots of (E) CcdB single-

site saturation mutagenesis library and (F) WT CcdB. 

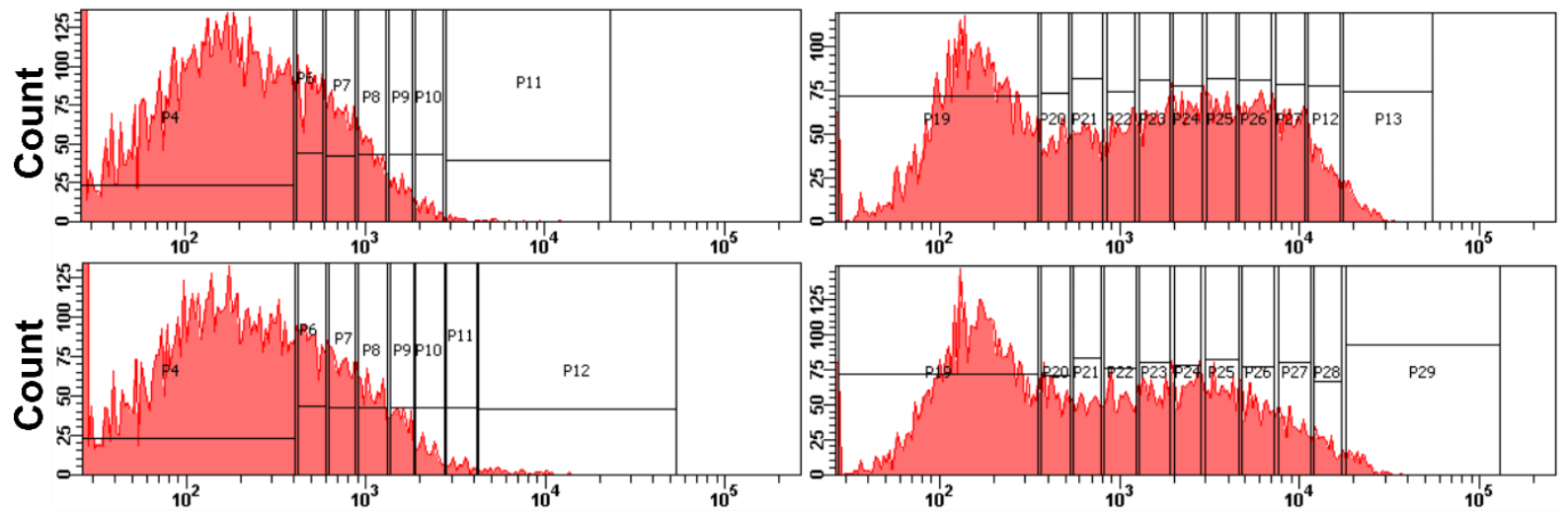

V18G

library
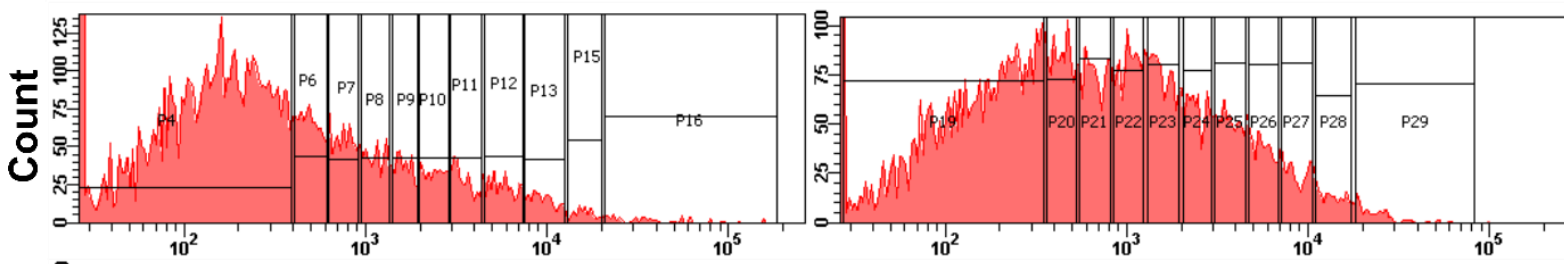

V20G

library
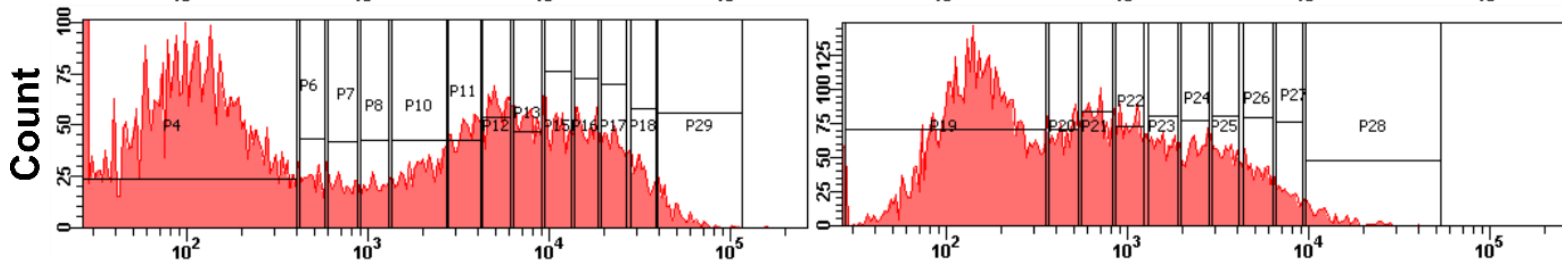

L36A

library

Supplementary Figure S2: Histograms of expression and binding to GyrA14 of PIM containing

CcdB libraries. Left and right panels show binding and expression histograms. Different populations sorted into bins based on expression and binding are indicated by the vertical lines. 


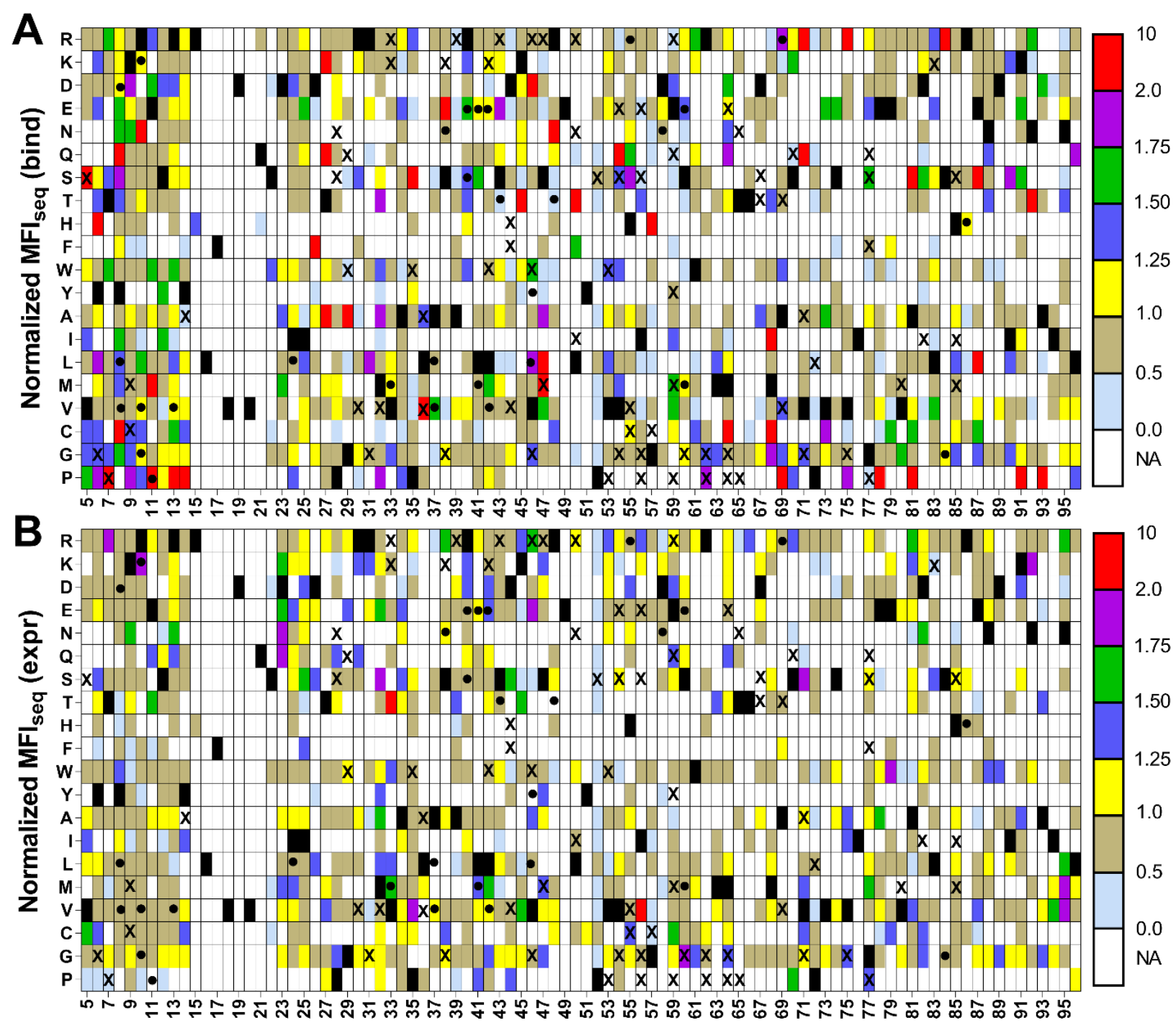

605 Supplementary Figure S3: Heat maps of normalized MFI $_{\text {seq }}$ (bind) and MFI $_{\text {seq }}$ (expr) for V18D

606 library. Normalized (with respect to V18D) values of $\mathrm{MFI}_{\text {seq }}$ (bind) (A) and $\mathrm{MFI}_{\text {seq }}$ (expr) (B) were 607 categorized in different ranges. Mutants with normalized $\mathrm{MFI}_{\text {seq }}$ (bind) or $\mathrm{MFI}_{\text {seq }}(\mathrm{expr})>1.25$ were 608 categorized as putative stabilized mutants. Black rectangle represents WT residue, mutants with no 609 data available are indicated with a white rectangle. Several single mutants were characterized in vitro 610 to estimate their stability, stabilized mutants having $\Delta \mathrm{T}_{\mathrm{m}}>1$ are indicated with a " $\bullet$ " and destabilized 611 mutants $\Delta \mathrm{T}_{\mathrm{m}}<0$ are indicated with an "X". 

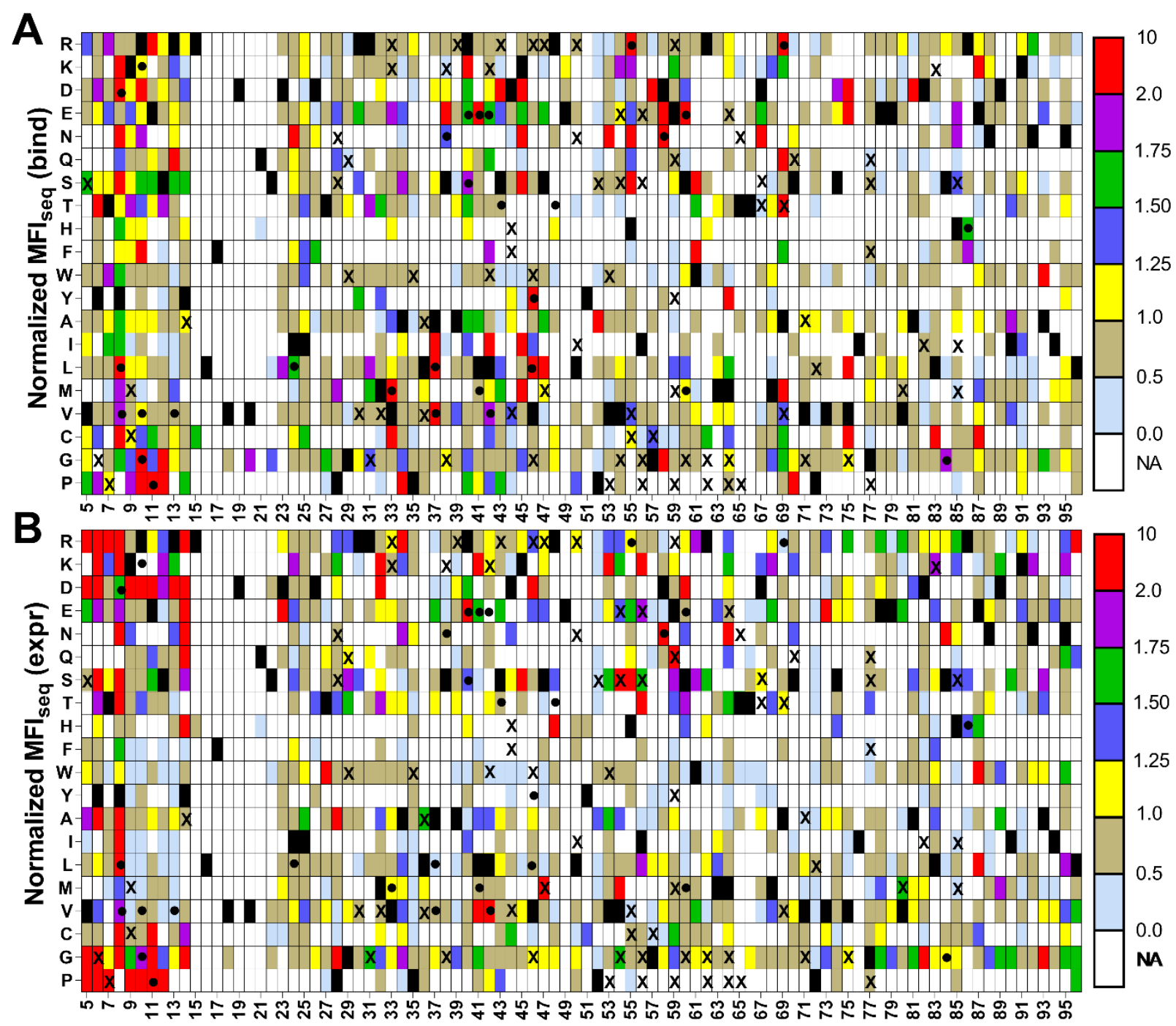

613 Supplementary Figure S4: Heat maps of normalized MFI $_{\text {seq }}$ (bind) and MFI seq $_{\text {(expr). Normalized }}$

614 (with respect to $\mathrm{V} 18 \mathrm{G}$ ) values of $\mathrm{MFI}_{\text {seq }}$ (bind) (A) and $\mathrm{MFI}_{\text {seq }}$ (expr) (B) were categorized in different 615 ranges. Mutants with normalized $\mathrm{MFI}_{\text {seq }}$ (bind) or $\mathrm{MFI}_{\text {seq }}$ (expr) $>1.25$ were categorized as putative 616 stabilized mutants. Black rectangle represents WT residue, mutants with no data available are indicated 617 with a white rectangle. Several single mutants were characterized in vitro to estimate their stability, 618 stabilized mutants $\left(\Delta \mathrm{T}_{\mathrm{m}}>1\right)$ are indicated with a "•" and destabilized mutants with $\left(\Delta \mathrm{T}_{\mathrm{m}}<0\right)$ are 619 indicated with an "X". 


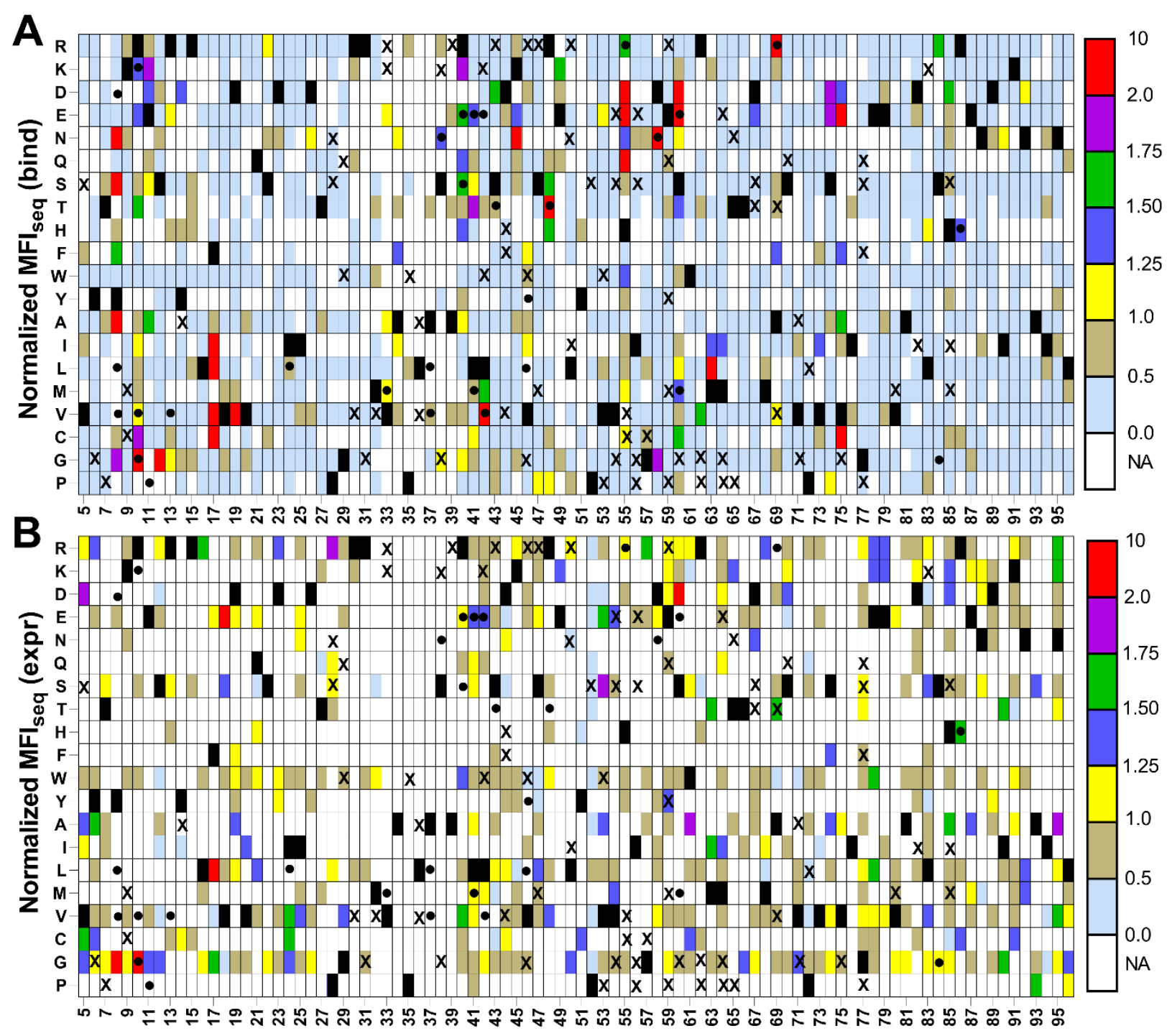

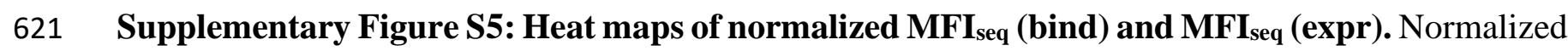

622 (with respect to $\mathrm{L}^{36 \mathrm{~A}}$ ) values of $\mathrm{MFI}_{\text {seq }}$ (bind) (A) and $\mathrm{MFI}_{\text {seq }}$ (expr) (B) were categorized in different 623 ranges. Mutants with normalized $\mathrm{MFI}_{\text {seq }}$ (bind) or $\mathrm{MFI}_{\text {seq }}$ (expr) $>1.25$ were categorized as putative 624 stabilized mutants. Black rectangle represents WT residue, mutants with no data available are indicated with a white rectangle. Several single mutants were characterized in vitro to estimate their stability, stabilized mutants $\left(\Delta \mathrm{T}_{\mathrm{m}}>1\right)$ are indicated with a "•" and destabilized mutants with $\left(\Delta \mathrm{T}_{\mathrm{m}}<0\right)$ are indicated with an "X". 


\section{A}

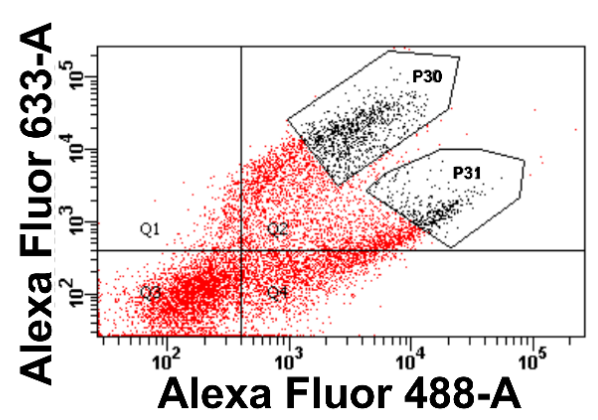

B

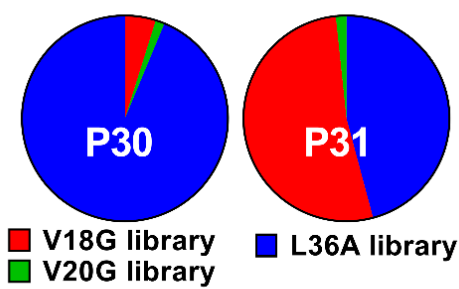

C

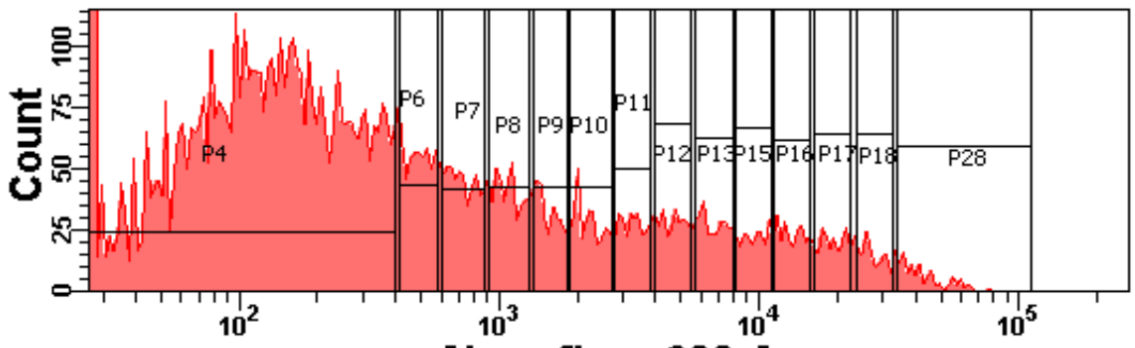

Alexa fluor 633-A

D

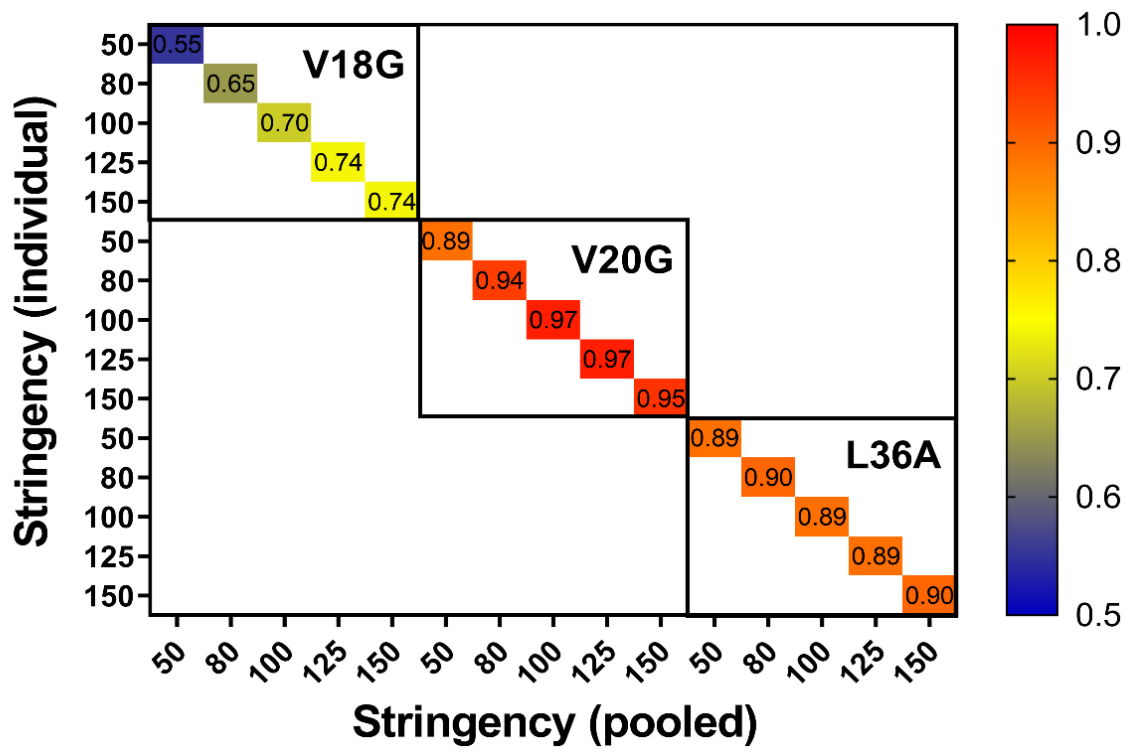

630 Supplementary Figure S6: FACS of pooled libraries. (A) Dot plot showing the expression and

631 binding of pooled library. Two different gates, P30 and P31 were used to sort the populations showing 632 the highest expression and binding. (B) Pie chart of relative enrichment of mutants from each library 633 after one round of sorting and deep sequencing in gates P30 and P31. (C) Sorting of pooled V18G, 634 V20G and L36A library based on binding to GyrA14. (D) Heat map of correlation coefficient between 635 the binding MFI of mutants calculated from an individual library and the pooled library at different 636 stringencies, where stringency is the minimum number of reads per mutant. 
637 Supplementary Table 1: Thermal stability of CcdB mutants estimated using TSA.

\begin{tabular}{|c|c|c|c|c|c|c|c|}
\hline Mutant & $\Delta \mathrm{T}_{\mathrm{m}}$ & Mutant & $\Delta \mathrm{T}_{\mathrm{m}}$ & Mutant & $\Delta \mathrm{T}_{\mathrm{m}}$ & Mutant & $\Delta \mathrm{T}_{\mathrm{m}}$ \\
\hline R62P & -24 & D67S & -5 & $\mathrm{P} 28 \mathrm{~N}$ & -1.5 & Y8L & 2 \\
\hline I56P & -17 & $\mathrm{I} 25 \mathrm{~A}$ & -4.5 & M32V & -1.5 & R10V & 2 \\
\hline $\mathrm{P} 35 \mathrm{~W}$ & -15.5 & $\mathrm{I} 25 \mathrm{~N}$ & -4.5 & $\mathrm{V46G}$ & -1.5 & I24L & 2 \\
\hline L50N & -15 & S43Y & -4 & G57C & -1.5 & I24Y & 2 \\
\hline V54S & -15 & V46R & -4 & E59Q & -1.5 & V33M & 2 \\
\hline P52S & -14.5 & S47R & -4 & A69V & -1.5 & E11P & 2.5 \\
\hline L50R & -14 & V53W & -4 & $\mathrm{V75G}$ & -1.5 & $\mathrm{R} 40 \mathrm{E}$ & 2.5 \\
\hline T7P & -13 & I25T & -3.5 & S12A & -0.5 & $\mathrm{~L} 42 \mathrm{E}$ & 2.5 \\
\hline D82I & -13 & S38K & -3.5 & A37D & -0.5 & S60M & 2.5 \\
\hline D67T & -11.5 & D44H & -3.5 & A37Y & -0.5 & $\mathrm{~L} 42 \mathrm{~V}$ & 3 \\
\hline L36V & -10 & R86E & -3.5 & S43A & -0.5 & $\mathrm{R} 48 \mathrm{~T}$ & 3 \\
\hline T65Q & -9.5 & Y14A & -3 & WT & 0 & $101 \mathrm{~N}$ & 3 \\
\hline D44F & -7 & $\mathrm{~L} 42 \mathrm{~K}$ & -3 & Y14W & 0 & A37V & 3.5 \\
\hline M64P & -7 & L50I & -3 & S47M & 0 & H55R & 3.5 \\
\hline G77P & -7 & D67L & -3 & K9R & 0.5 & S84G & 3.5 \\
\hline D44V & -6.5 & K9M & -2.5 & K45Y & 0.5 & Y8D & 4 \\
\hline P72L & -6.5 & D26S & -2.5 & V46W & 0.5 & $\mathrm{I} 24 \mathrm{~K}$ & 4 \\
\hline R86D & -6.5 & R30V & -2.5 & $\mathrm{R} 48 \mathrm{G}$ & 0.5 & V46L & 4 \\
\hline A39R & -6 & S38G & -2.5 & A69T & 0.5 & $\mathrm{R} 86 \mathrm{H}$ & 4 \\
\hline E59P & -6 & L42W & -2.5 & R86K & 0.5 & S38N & 4.5 \\
\hline V71A & -6 & R62G & -2.5 & Y8V & 1 & R40S & 4.5 \\
\hline G77S & -6 & H55C & -2 & R13V & 1 & D58N & 4.5 \\
\hline P28S & -5.5 & E59R & -2 & $\mathrm{~L} 41 \mathrm{M}$ & 1 & A69R & 4.5 \\
\hline V53P & -5.5 & S60G & -2 & S43T & 1 & L41E & 5 \\
\hline E59Y & -5.5 & S70Q & -2 & R10K & 1.5 & S60E & 7.5 \\
\hline E79I & -5.5 & K9C & -1.5 & I24E & 1.5 & R10G & 10 \\
\hline D26A & -5 & R13F & -1.5 & A37L & 1.5 & & \\
\hline $\mathrm{P} 28 \mathrm{Y}$ & -5 & D26V & -1.5 & V46Y & 1.5 & & \\
\hline
\end{tabular}

